\title{
A randomized, double-blind, placebo-controlled trial of anti-IgE for chronic rhinosinusitis*
}

\author{
J.M. Pinto, N. Mehta, M. DeTineo, J. Wang, F.M. Baroody, R.M. Naclerio \\ Department of Surgery, Section of Otolaryngology-Head and Neck Surgery, The University of Chicago, \\ Chicago, IL, USA
}

\begin{abstract}
SUMMARY Evidence suggests IgE may play a role in chronic rhinosinusitis (CRS). We sought to determine if treatment with a monoclonal antibody against IgE (omalizumab) is effective in reducing CRS inflammation.

We performed a randomized, double blind, placebo controlled clinical trial in subjects with CRS despite treatment (including surgery). Subjects were randomized to receive omalizumab or placebo for 6 months. The primary outcome was quantitative measurement of sinus inflammation on imaging. Secondary outcome measures included quality of life, symptoms, and cellular inflammation, nasal airflow (NPIF) and olfactory testing (UPSIT).

Subjects on omalizumab showed reduced inflammation on imaging after treatment, whereas those on placebo showed no change. The net difference, however, was not different between treatments. Treatment with omalizumab was associated with improvement in the Sino-Nasal Outcome Test (SNOT-20) at 3, 5, and 6 months compared to baseline with no significant changes in the control group. Remaining measures showed no significant differences across treatments.

We conclude that IgE plays, at most, a small role in the mucosal inflammation of CRS and the symptoms. Placebo controlled, blinded studies with larger enrollment are needed to determine the clinical significance of any potential change.
\end{abstract}

Key words: chronic rhinosinusitis, Xolair, omalizumab, anti-IgE, inflammation, randomized clinical trial, allergy

\section{INTRODUCTION}

The hallmark of CRS is sinus mucosal inflammation, a parameter thought to correlate with disease severity. Therapies that address the genesis of the dysregulated mucosal immune response and resultant chronic inflammatory state in the sinuses are needed. To date there is a paucity of data on effective therapies for severe disease, especially using randomized trial designs.

Some of the inflammatory features of CRS are similar to those observed in allergic asthma. Asthma and allergic rhinitis (AR) are two diseases strongly associated with CRS. Previous studies have suggested that allergies are an underlying factor in 40 $94 \%$ of patients with $\mathrm{CRS}^{(1,2)}$, though the exact relationship remains controversial, with cause and effect unclear. Similarly, there is a strong epidemiological association between asthma and CRS. A vast majority of moderate-to-severe asthmatics have abnormal findings on sinus imaging ${ }^{(3)}$, and there is a correlation between the severity of asthma and the number of mucosal abnormalities on CT scans ${ }^{(3)}$. Tissue samples from patients with CRS with nasal polyposis show abundant eosinophils and a Th2 cytokine profile, both hallmarks of the lower airway inflammation found in patients with asthma. These data suggest that similar pathological mechanisms may be involved in these diseases and that treatment strategies might target common mechanistic pathways.

$\mathrm{IgE}$, a key mediator in the inflammatory process of $\mathrm{AR}$ and allergic asthma, has been implicated in CRS. Serum levels of this immunoglobulin have been shown to correlate with the severity of mucosal disease measured by CT imaging ${ }^{(4)}$. Some data suggest a role for local elevation of $\operatorname{IgE}$ in sinus tissue ${ }^{(5)}$. Hence, IgE is an attractive target for therapeutic intervention in CRS.

Omalizumab (Xolair ${ }^{\circledR}$ ) is an anti-IgE monoclonal antibody approved for the treatment of moderate and severe persistent allergic asthma. Omalizumab reduces the levels of $\operatorname{IgE}$ in the serum and tissues, thereby blocking the IgE-mediated inflammatory cascade. This anti-IgE treatment has also been shown 
to effectively treat AR. Furthermore, limited uncontrolled data to date suggest that this treatment might be useful in CRS ${ }^{(6,7)}$. We therefore hypothesized that anti-IgE treatment would decrease sinonasal inflammation and improve symptoms and related measures in CRS, and thus may be a novel and useful treatment option for patients with CRS.

\section{MATERIALS AND METHODS}

Subjects

Males and females 18 - 75 years of age with CRS were recruited in Chicago from 2004 to 2007. CRS was defined by symptoms (nasal obstruction, nasal discharge, facial pain, hyposmia) for greater than 12 weeks, confirmatory findings on nasal endoscopy, and evidence of inflammation on sinus CT scan (13). Serum total $\mathrm{IgE}$ between $30-700 \mathrm{IU} / \mathrm{ml}$ was also required because this is the level for which the drug is approved for the treatment of asthma. We excluded subjects weighing $>150 \mathrm{~kg}$, those with contraindications to omalizumab, and subjects with secondary causes of CRS (immunocompromise, genetic disease). Though we set no limits on disease severity, the majority of subjects had severe and refractory disease; for example, all had undergone endoscopic sinus surgery and the majority presented with nasal polyposis $(7 / 7$ in the omalizumab group and 5/7 in the placebo group).

\section{Study protocol}

We performed a randomized, placebo-controlled double-blind study examining the effects of anti-IgE in CRS. During the screening visit, medical records were reviewed to confirm entry criteria. A CT scan was performed to document the state of sinus mucosal inflammation at study outset. Serum IgE levels were obtained.

At enrollment (visit 1, baseline), symptoms were recorded and general (Short Form-36 ${ }^{(8)}$ [SF-36]) and disease-specific (Sinonasal Outcome Test-20 ${ }^{(9)}$ [SNOT-20]) quality of life measured. Objective assessment was obtained using nasal endoscopic examination to score polyp size on a scale of 0 - 4 using published criteria ${ }^{(10)}$. Nasal lavage for eosinophil counts ${ }^{(11)}$, olfactory testing (the University of Pennsylvania Smell Identification Test [UPSIT]) ${ }^{(12)}$, and nasal peak inspiratory flow (NPIF, performed in triplicate and averaged) were also obtained. Subjects were then randomized to omalizumab or placebo groups.

At enrollment and every 4 weeks for the 6 month duration of the study, patients received either omalizumab $(0.016 \mathrm{mg} / \mathrm{kg}$ per IU total serum IgE/mL subcutaneously) or placebo injection. Since omalizumab is dosed by weight, some subjects required bimonthly dosing according to standard asthma dosing guidelines $(0.016 \mathrm{mg} / \mathrm{kg})$. Just prior to dosing at each follow-up visit, quality of life measures, nasal lavage, nasal endoscopy, and NPIF were repeated. For subjects dosed bimonthly, measures were obtained only once a month.
Throughout the trial, subjects recorded symptoms daily (nasal obstruction, nasal discharge, facial pain, and hyposmia) each recorded on a 4 point scale $[0=$ none, $1=$ mild, $2=$ moderate, 3 = severe]; total scores were summed for a Total Nasal Symptom Score [TNSS]). At the 6 month visit, a final sinus CT scan and a final UPSIT were repeated. This study was approved by our IRB and the FDA. It was registered with clinicaltrials.gov prior to initiation (NCT00117611).

\section{Safety}

Patients were monitored after each injection based on prevailing guidelines. These changed during the study to the current recommendation which is 2 hours of observation following the first 3 injections due to new FDA warnings regarding the possible risk of anaphylaxis (http://www.fda.gov/cder/drug/ infopage/omalizumab/default.htm). This requirement ended recruitment because of the time commitment required for participation in the study by volunteers. To comply with current FDA and society recommendations, patients were also given an Epipen to take home.

\section{Outcome measures}

The primary outcome measure for this study was sinus inflammation as determined by CT imaging. For this assessment, a standard pre-treatment and post-treatment coronal CT image was selected at the osteomeatal unit (OMU). Pre- and posttreatment images were aligned (Figure 1a).
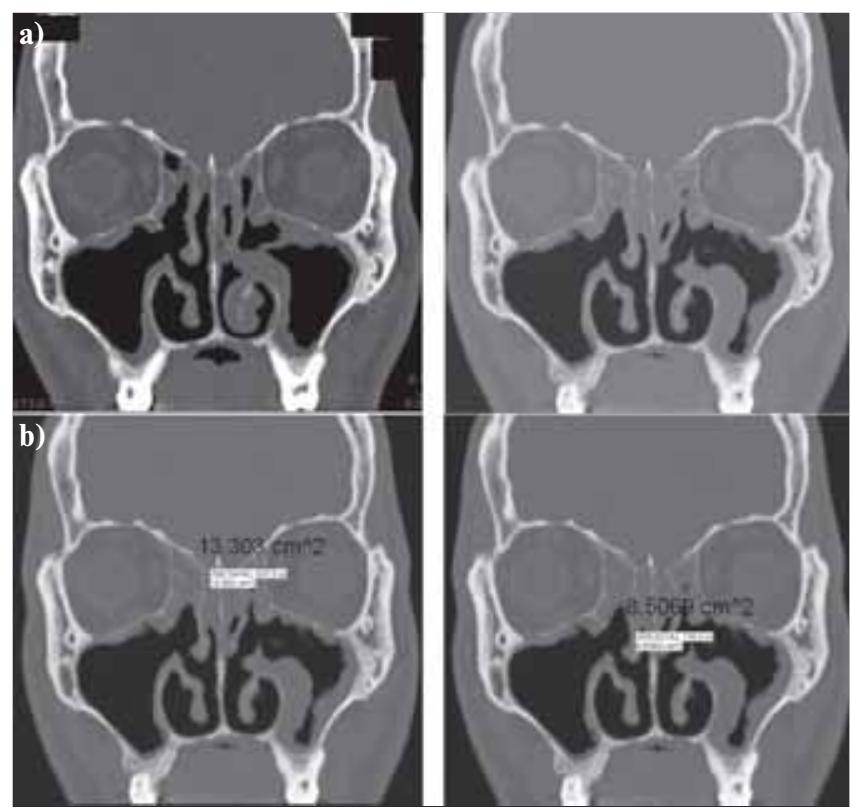

Figure 1. a) Aligned Coronal CT Slices. (left) Pre-treatment coronal CT slice showing the maxillary and ethmoid sinuses at the OMU (right) Aligned post treatment slice correlating to pre treatment slice. b) Measurement of Sinus Opacification. (left) Bony outline of sinuses at the OMU is selected with the corresponding area measurement given by the software (Stentor) (right) Outline of the air within the passage is selected with corresponding area measurement given. Using these measurements, percent inflammation is determined. 
Table 1. Demographics of each group.

\begin{tabular}{lccc}
\hline & omalizumab & placebo & p-value \\
\hline Subjects & 7 & 7 & \\
Demographics & & & \\
$\quad \%$ Male (n/N) & $43 \%(3 / 7)$ & $100 \%(7 / 7)$ & $<0.02$ \\
$\quad$ Age (mean \pm SD) & $43.1 \pm 9.8$ & $48.6 \pm 9.1$ & $<0.30$ \\
Medication Use & & & \\
$\quad$ (\%/n/N) & & & \\
Intranasal steroids & $0.714(4 / 7)$ & $0.714(5 / 7)$ & $<0.59$ \\
Antihistamines & $0.142(1 / 7)$ & $0.571(4 / 7)$ & $<0.27$ \\
Antileukotrienes & $0.571(4 / 7)$ & $0.571(4 / 7)$ & $<1.00$ \\
Systemic steroids & $0.428(3 / 7)$ & $0.000(0 / 7)$ & $<0.19$ \\
Inhaled Asthma Therapy & $0.716(5 / 7)$ & $0.428(3 / 7)$ & $<0.59$ \\
History of Immunotherapy & $0.000(0 / 7)$ & $0.285(2 / 7)$ & $<0.46$ \\
Quality of Life & & & \\
SNOT-20 (mean \pm sem) & $45.7 \pm 5.6$ & $46.0 \pm 11$ & $<0.877$ \\
SF-36 (domains mean \pm sem) & $61.8 \pm 15.2$ & $67.6 \pm 10.5$ & $<0.418$ \\
& & & \\
NPIF (mean, range) & $93.3(53.3-$ & $133.3(113.3-$ & $<0.303$ \\
& $173.3)$ & $156.7)$ & \\
Nasal Lavage Eosinophils & & & \\
(\% \pm sem) & $7.6 \pm 2.2$ & $12.6 \pm 6.5$ & $<0.715$ \\
Nasal Endoscopy score & & & \\
(median, range) & $1.5(0.5-3)$ & $1.25(0-2.5)$ & $<0.373$ \\
UPSIT (mean \pm sem) & $13 \pm 2.8$ & $19 \pm 3.4$ & $<0.174$ \\
\% Opacification Baseline CT & & & \\
(mean \pm sem) & $76.0 \% \pm 6.9$ & $75.9 \% \pm 6.1$ & $<0.568 *$ \\
\hline
\end{tabular}

Using tools on our web-based hospital radiology software (Stentor, Phillips), we outlined the bony limits of the maxillary, ethmoid, and frontal sinuses to obtain a total sinus area on the aligned slices (Figure 1b). Slices were aligned to match pre- and post-treatment using bony landmarks. We then repeated this measurement selecting only the air filled portions of the same area. Using the area measurement of the air and of the total area of the sinus, the percent inflammation (non-air occupied sinus surface) in the standard coronal slice was calculated (Figure 1b). A similar method has been described by others ${ }^{(13)}$. All CT scan were read blinded to treatment category.

General (SF-36) and specific quality of life (SNOT-20), nasal airflow (NPIF), olfactory function (UPSIT), symptoms, nasal endoscopy scores, and inflammation in nasal lavage (eosinophils) were assessed as secondary outcome measures using standard methodologies (see above).

\section{Statistical analysis}

Repeated measures were first evaluated by analysis of variance (ANOVA [parametric measures] or Friedman [nonparametric measures]) followed by post-hoc testing if appropriate. Quality of life measures were all normally distributed and were analyzed with parametric statistics using the t-test with means reported. Changes between pre- and post-treatment were compared using a paired t-test. Between group comparisons were made by a non-paired t-test. Percentage of inflammation on imaging, UPSIT scores, eosinophil counts in nasal lavage, nasal endoscopy scores, NPIF and symptoms scores were not normal- ly distributed and therefore were analyzed using nonparametric tests. Paired analysis was done with the Wilcoxon signed-rank test. Unpaired analysis was done with the Mann Whitney U test. $\mathrm{P}$ values $<0.05$ were considered significant for all analyses.

\section{RESULTS}

Enrollment

We viewed this as a pilot study and asked our IRB for permission to enroll 25 subjects in each group. After initial success, we began having difficulty recruiting subjects. The primary reason was the reluctance of eligible subjects to enroll in a trial

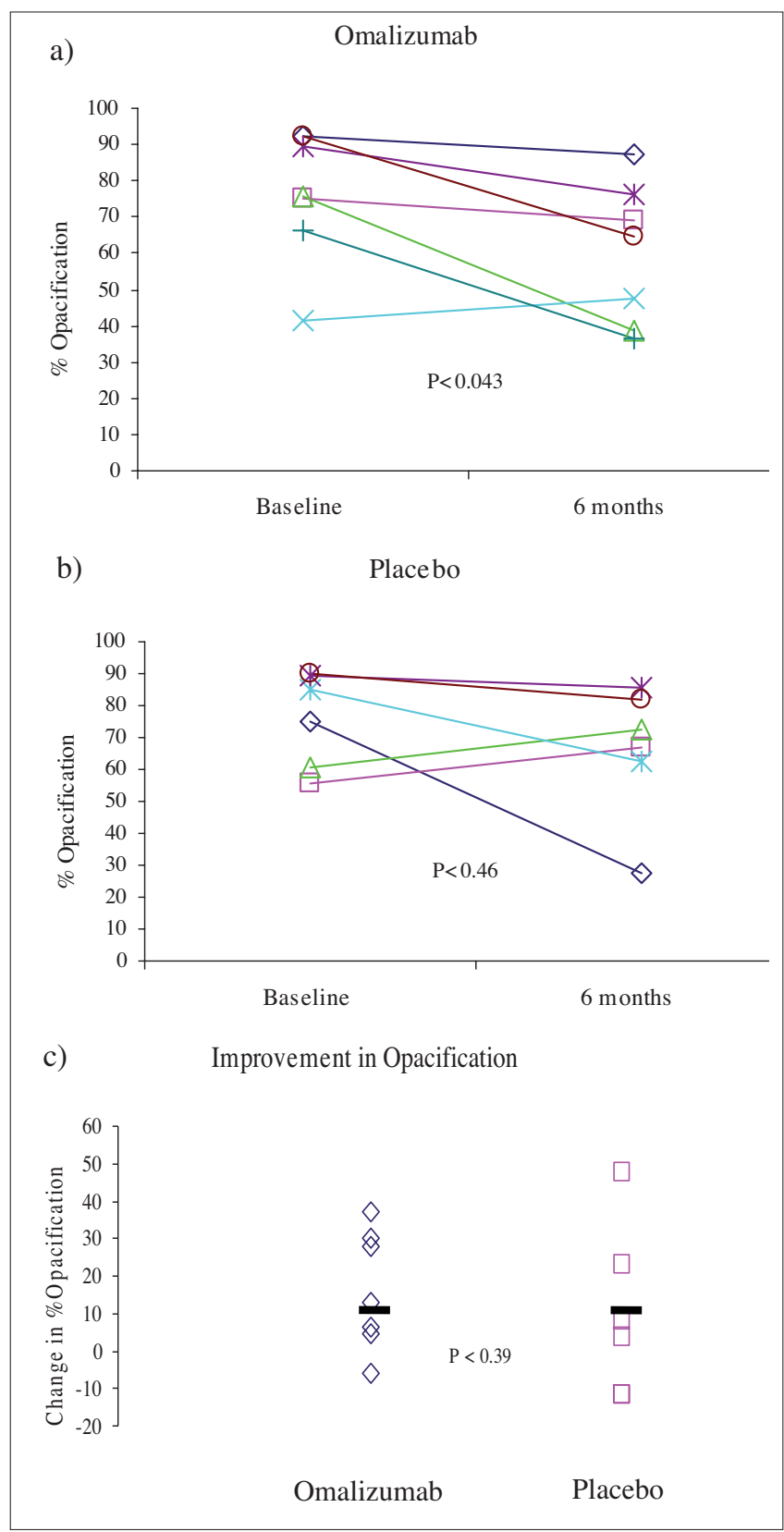

Figure 2. Sinus CT opacification a) Omalizumab. b) Placebo. Note, $n=$ 6 in the placebo group due to missing data secondary to technical problems with image analysis from outside hospital CT scan in one patient. c) Improvement in sinus opacification by $\mathrm{CT}$ across trial for each treatment. 
with risk of receiving placebo treatment and time commitment (7 visits). Other impediments included the need in some subjects to have twice monthly dosing due to weight, IgE levels being too low or high to qualify and negative allergy tests. To circumvent these obstacles to recruitment, we then attempted to recruit subjects at other sites. Unfortunately, in over 18 months, no external site $(n=6)$ enrolled any subjects. Around this time, the FDA issued a warning about anaphylaxis, leading to further delays in amending the protocols to be compliant with guidelines. With our inability to enroll more subjects, we terminated the trial.

We report here the results of 14 patients with 7 in each treatment group who completed the study. There were 10 males and 4 females (Table 1) with no dropouts. There were more men in the placebo group than in the treatment group. The subjects in both treatment groups were well matched for use of sinusitis medications (Table 1) and initial disease as measured by \% sinus opacification on baseline CT images (Table 1). Subjects were randomized and followed throughout the trial in a blinded fashion.

\section{Safety}

No side effects or adverse events occurred during the study.

\section{Effect of omalizumab on sinus inflammation}

A comparison of the change in percent opacification across treatment from matched coronal CT slices at the OMU for each patient was performed (Figure 2). A statistically significant reduction in inflammation was found for the omalizumab treatment group from baseline to conclusion of the study (median pre $76.1 \%$, post $60.0 \%$; $<0.043$, Figure $2 a$ ) with no significant change in the placebo group (median pre $75.9 \%$, post $66.1 \%$, p < 0.463 , Figure 2b). However, comparing the magnitude of change (pre-treatment minus post-treatment) across treatment groups, there was no significant difference (median change omalizumab 11.9\%, placebo $5.9 \%$; p $<0.391$; Figure 2c).

\section{Secondary outcomes}

\section{Quality of Life}

Baseline mean SNOT-20 scores between 2 and 3 were consistent with prior studies of CRS patients ${ }^{(14)}$. There seemed to be a significant placebo effect with a trend toward improved quality of life from enrollment to the first month of treatment in both groups (Figure 3a). Comparing across groups, we summed the differences from baseline for each subject and treatment (1st month treatment minus baseline plus the $2^{\text {nd }}$ month treatment minus baseline) and there was no significant net difference across treatments (median omalizumab -5.5, placebo $-2.3, \mathrm{p}<0.60$ ) (Figure $3 \mathrm{~b}$ ). Interestingly, the median change in SNOT-20 scores across the study (last visit minus baseline) was consistent with a clinically significant improvement (defined as at least 0.8 ) in the omalizumab group (-1.05) and no clinically significant change in the placebo group

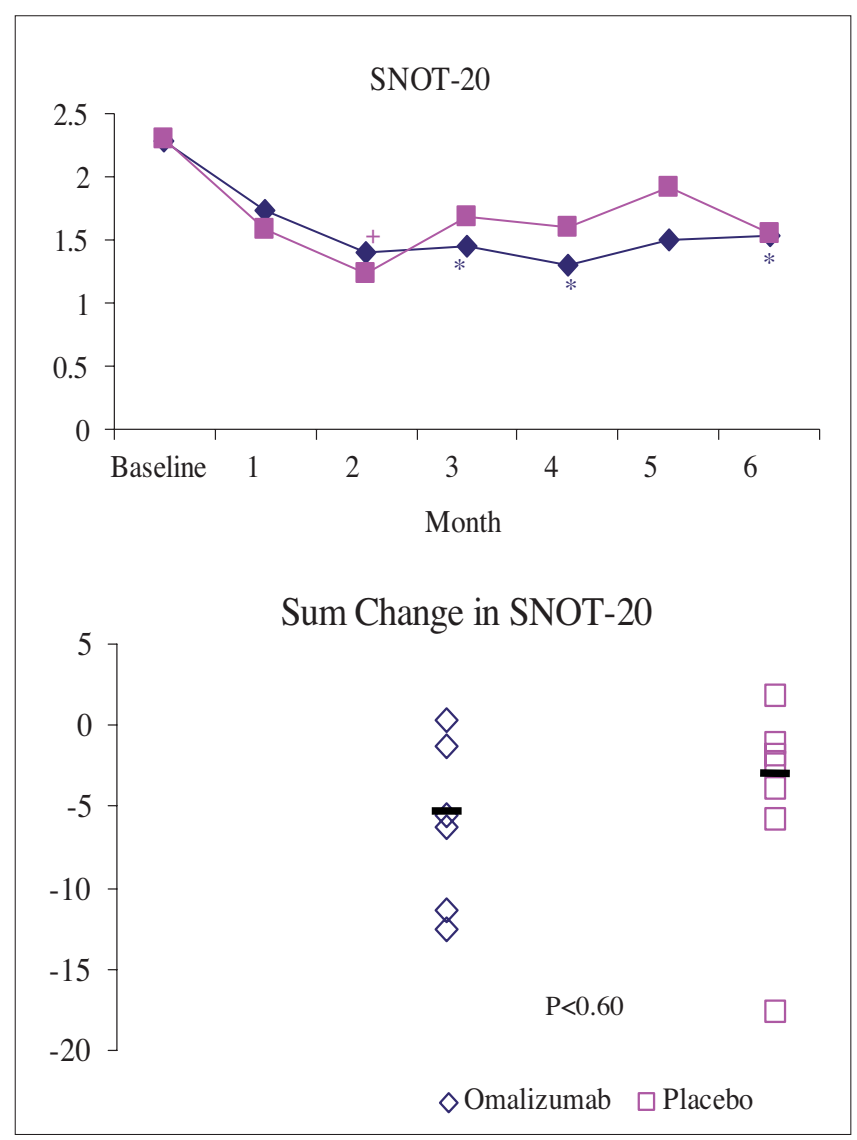

Figure 3. SNOT-20. (top) Score at baseline and monthly throughout the study. (bottom) Sum of change in scores (each visit minus baseline) across the study. ${ }^{*},+$ : $p<0.05$.

$(-0.20)$, but there was no difference in the net magnitude of this change between the groups ( $\mathrm{p}<0.78$ ).

Baseline mean domain score for the SF-36 showed that both groups had lower quality of life than normal subjects consistent with prior studies ${ }^{(15)}$. There were no significant differences within treatments for any domain $(\mathrm{p}>0.05$, all comparisons). Across treatments, there were also no significant differences ( $\mathrm{p}>0.05$, all comparisons) except for one domain, vitality (omalizumab 9.4, placebo 12.5, $\mathrm{p}<0.05$ ).

\section{Olfaction}

The UPSIT was used to gauge olfactory function with higher scores corresponding to a better sense of smell. Both active and placebo groups showed improvement in total UPSIT score (data not shown). Comparing across groups, the net change in total UPSIT score was not statistically different (omalizumab 3, placebo $4, \mathrm{p}<0.31)$.

\section{Nasal endoscopy scores}

There were no significant changes within in endoscopy scores for either group (data not shown). Net change across treatments were not significantly different (omalizumab 0 , placebo $-0.5, \mathrm{p}<0.58)$. 


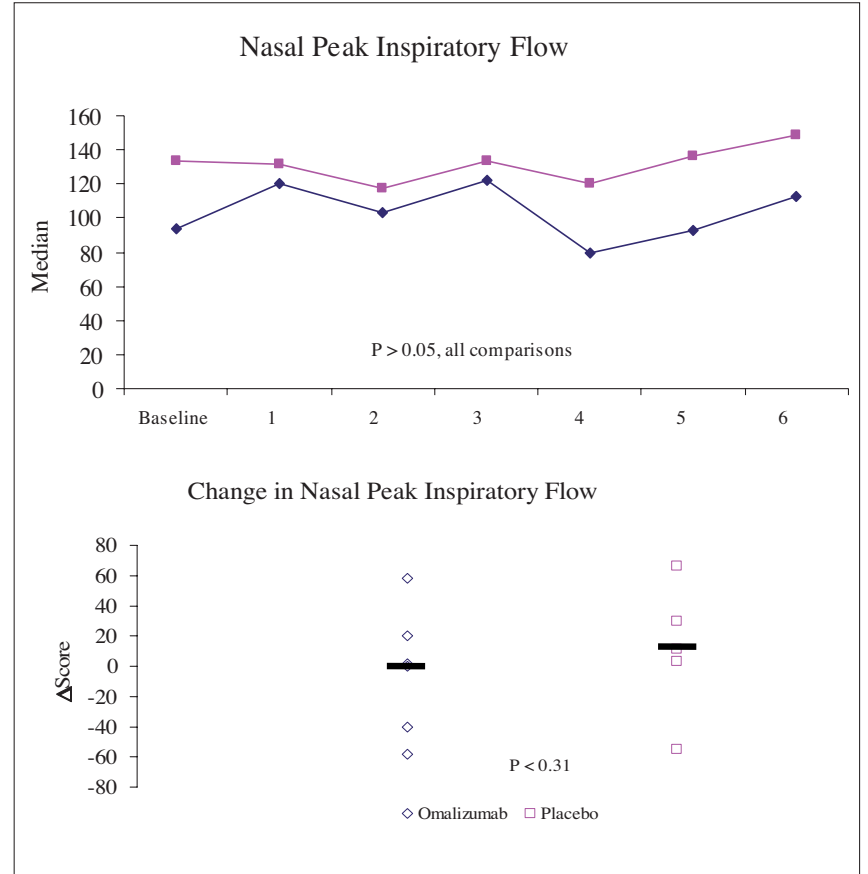

Figure 4. Nasal Peak Inspiratory Flow. (top) NPIF at baseline and monthly throughout the study (median). (bottom) Change in NPIF from 6 month visit compared to baseline.

\section{Eosinophils in nasal lavage}

Inflammation was assessed in nasal lavage using eosinophil counts. Analysis of variance showed no significant difference in either treatment ( $p>0.05$, all comparisons; data not shown). There were no differences in net change between the groups (median change in $\%$ : omalizumab -2 , placebo -9 , p $<0.47$ ).

\section{NPIF}

There were no statistically significant differences in NPIF during any of the visits for within ( $p>0.05$, all comparisons) or net change across groups (median: omalizumab -3.1 , placebo $11.3, \mathrm{p}<0.31$ ) (Figure 4). Ranges of this measure and variability were consistent with other studies ${ }^{(16)}$.

\section{Sinonasal symptoms}

The median TNSS for each month did not vary between visits by analysis of variance for either group ( $p>0.05$, all comparisons), with no significant net difference across treatments (omalizumab -1 , placebo $0, \mathrm{p}<0.21$ ).

\section{Baseline medication use and use rescue medications}

Baseline medications were not different at the outset (Table 1). Since we allowed use of rescue medications during the trial, we then compared the reported use of courses of systemic steroids, antibiotics, and added adjunctive medications (antileukotrienes, antihistamines, or intranasal steroids). There were few differences between the groups, though small numbers preclude definitive analysis. Subjects on omalizumab used fewer courses of steroids over the trial (median 0) compared to placebo

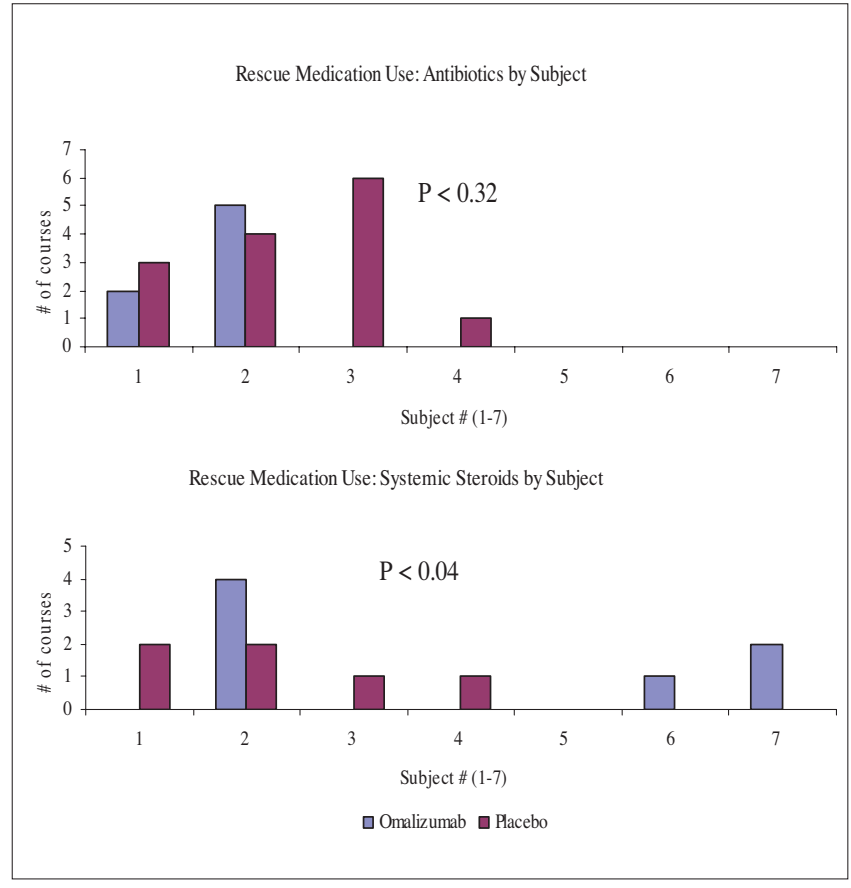

Figure 5. Rescue Medication Use by Subject. (top) Antibiotic courses. Median omalizumab 0, placebo $1, \mathrm{p}<0.32$. (bottom) Systemic steroid courses. Median omalizumab 0, placebo $1, \mathrm{p}<0.043$ ).

(median 1) $(\mathrm{p}<0.043)$. There also seemed to be a trend toward less use of antibiotics in the omalizumab group (median omalizumab 0, placebo $1, \mathrm{p}<0.32$ ) (Figure 5). There was no discernible pattern among users of systemic steroids and improvement on imaging nor did responding subjects seem to have used rescue medications closer to the end of the study.

\section{DISCUSSION}

Our results suggest that omalizumab may reduce sinonasal inflammation assessed by imaging, perhaps providing support for a role for IgE in the inflammatory process of CRS. This difference, about $10 \%$, was small and did not translate into any measurable symptomatic or physiological benefit for our patients. Doing a larger study would not be expected to change the magnitude of the difference and the clinical significance of this change remains unclear. Given the standard deviation of the net change in the omalizumab subjects [ 16] and this net difference [10], a sample of 42 subjects in each group would be required to demonstrate a treatment effect with $80 \%$ power at a significance level of 0.05 ( 2 sided).

We used a novel method of quantifying sinus inflammation to more closely capture a subtle change that might occur with a therapeutic intervention, especially in severe disease. Other methods that are used to stage disease are crude in their quantification (e.g., clear, partially opacified, completely opacified) and were not designed to assess changes in disease over time. It should also be noted that imaging findings and symptoms have yet to be found to correlate due to a variety of possible 
reasons ${ }^{(17)}$, though a closer correlation was found when examining these relationships in response to medical treatment ${ }^{(18)}$. We chose CT image analysis as a primary outcome measure to answer the hypothesis that anti-IgE would decrease mucosal inflammation. We also examined a number of other clinical measures and symptoms. Our study failed to show any notable improvement in any of these disease parameters.

To our surprise, the best improvement of CT inflammation occurred in a subject who received placebo treatment, a fact that emphasizes the variability in the natural history of the disease. Moreover, this emphasizes the critical need for randomized trials to determine treatment efficacy in this disease.

A major conclusion of this study is that performing randomized, placebo controlled clinical trials of prolonged therapies for chronic sinusitis is extremely challenging. First, we faced significant recruitment challenges despite the busy rhinology practices of several rhinologists and general otolaryngologists at a major, urban, academic medical center as well as local and national advertising and recruitment at multiple centers. Despite our best efforts, there were only 14 patients who completed the study. We faced significant challenges recruiting subjects for this trial, with many patients unwilling to enroll due to the time and logistical requirements mandated by monthly visits, the possibility of receiving placebo therapy and, possibly, due to small but well publicized risks of adverse reactions to omalizumab, FDA black box warnings, and change in guidelines lengthening visits and delays awaiting revised IRB approvals.

This trial faced a number of challenges in design and implementation. All of our patients had had surgery and presented with severe inflammation. Hence, this group may present a form of severe, irreversible disease that responds poorly to any treatment, thereby limiting our ability to see an effect. A subject population with milder, perhaps more reversible inflammatory disease may offer us the ability to detect a greater effect. We opted to allow subjects to continue their current sinonasal medication regimen and used rescue medications as deemed appropriate by the clinician. In essence, we performed an addon study, which may have required a greater impact on disease measures to show a significant difference between treatments. Though there were no significant differences in the number or classes of medications at baseline, subjects were heavily medicated related to the severity of their disease. There may have been subtle differences in anti-inflammatory therapy between groups that clouded our outcomes. Moreover, there was substantial use of rescue medication therapy, including systemic steroid treatments, which may influence our results. However, had we precluded rescue therapy or limited medication use, we believe recruitment would have been even more difficult and challenged our commitment to our providing optimal care for our patients. Future trials will have to consider creative methods to overcome these barriers, perhaps by standardizing therapy for all subjects or using a withdrawal design.
Aside from efficacy, anti-IgE therapy faces other hurdles before it would be considered for use in CRS, including expense, the requirement for long clinic visits for administration, adverse events, and lack of potential utility in subjects without elevated IgE. Our largely negative results emphasize that CRS remains an extremely heterogeneous disease, likely with many triggers, multiple and overlapping inflammatory pathways, and diverse etiologies (fungus, enterotoxins, environmental pollutants, genetic predisposition, etc.) and has a variable natural history. This heterogeneity may require subclassification and targeted therapies for different types to achieve improvement. Indeed, there is some data to suggest that there are different forms of inflammation in some forms of CRS. However, one must consider that refractory CRS is challenging to treat, frequently requires expensive testing and therapies, some with unproven benefit (e.g., nebulized medications). Moreover, the societal costs are enormous when one considers decreased productivity, absenteeism, and other burdens of this disease. Hence, active search for new treatments or redirection of therapies in use for other conditions remains a high priority in this field.

In summary, our pilot study demonstrates at most a small, clinically irrelevant effect on CRS. Larger studies may provide additional data on the role of anti-IgE therapy for CRS.

\section{ACKNOWLEDGEMENTS}

Supported in part by a grant from Genentech and the McHugh Otolaryngology Research Fund. JMP was supported by a Dennis W. Jahnigen Career Development Award from the American Geriatrics Society. The study described in this manuscript was approved by the IRB of The University of Chicago. It was registered at Clinicaltrials.gov (\#NCT00117611). The investigators had full access to all the data in the study and JMP takes responsibility for the integrity of the data and the accuracy of the data analysis.

\section{ABBREVIATIONS}

Chronic rhinosinusitis (CRS), nasal peak inspiratory flow (NPIF), total nasal symptom score (TNSS), University of Pennsylvania Smell Identification Test (UPSIT), Sino-Nasal Outcome Test (SNOT-20), Short Form-36 (SF-36), osteomeatal unit (OMU).

\section{REFERENCES}

1. Benninger MS. Rhinitis, Sinusitis, and Their Relationships to Allergies. Am J Rhinology 1992; 6: 37-43.

2. Van Dishoeck HA, Franssen MG. The incidence and correlation of allergy and chronic maxillary sinusitis. Pract Otorhinolaryngol (Basel) 1957; 19: 502-506.

3. Bresciani M, Paradis L, Des Roches A, et al. Rhinosinusitis in severe asthma. J Allergy Clin Immunol 2001; 107: 73-80.

4. Baroody FM, Suh SH, Naclerio RM. Total IgE serum levels correlate with sinus mucosal thickness on computerized tomography scans. J Allergy Clin Immunol 1997; 100: 563-568. 
5. Bachert C, Zhang N, Patou J, et al. Role of staphylococcal superantigens in upper airway disease. Curr Opin Allergy Clin Immunol 2008; 8: 34-38.

6. Grundmann SA, Hemfort PB, Luger TA, et al. Anti-IgE (omalizumab): a new therapeutic approach for chronic rhinosinusitis. $\mathrm{J}$ Allergy Clin Immunol 2008; 121: 257-258.

7. Menendez R. Omalizumab Induces Resolution of Fungal Sinusitis in a 15 Year Old Asthmatic Patient. J Allergy Clin Immunol 2006; 117: S8.

8. Jenkinson C, Coulter A, Wright L. Short form 36 (SF36) health survey questionnaire: normative data for adults of working age. BMJ 1993; 306: 1437-1440.

9. Piccirillo JF, Merritt MG, Jr., Richards ML. Psychometric and clinimetric validity of the 20-Item Sino-Nasal Outcome Test (SNOT-20). Otolaryngol Head Neck Surg 2002; 126: 41-47.

10. Malm L. Assessment and staging of nasal polyposis. Acta Otolaryngol 1997; 117: 465-467.

11. Saengpanich S, Assanasen P, deTineo M, et al. Effects of intranasal azelastine on the response to nasal allergen challenge. Laryngoscope 2002; 112: 47-52.

12. Doty RL, Shaman P, Kimmelman CP, et al. University of Pennsylvania Smell Identification Test: a rapid quantitative olfactory function test for the clinic. Laryngoscope 1984; 94: 176-178.

13. Ponikau JU, Sherris DA, Weaver A, et al. Treatment of chronic rhinosinusitis with intranasal amphotericin B: a randomized, placebo-controlled, double-blind pilot trial. J Allergy Clin Immunol 2005; 115: 125-131.

14. Wallwork B, Coman W, Mackay-Sim A, et al. A double-blind, randomized, placebo-controlled trial of macrolide in the treatment of chronic rhinosinusitis. Laryngoscope 2006; 116: 189-193.
15. Gliklich RE, Metson R. The health impact of chronic sinusitis in patients seeking otolaryngologic care. Otolaryngol Head Neck Surg 1995; 113: 104-109.

16. Ebbens FA, Scadding GK, Badia L, et al. Amphotericin B nasal lavages: not a solution for patients with chronic rhinosinusitis. J Allergy Clin Immunol 2006; 118: 1149-1156.

17. Bhattacharyya N. Relationship between mucosal inflammation, computed tomography, and symptomatology in chronic rhinosinusitis without polyposis. Ann Otol Rhinol Laryngol 2008; 117: 517-522.

18. Subramanian HN, Schechtman KB, Hamilos DL. A retrospective analysis of treatment outcomes and time to relapse after intensive medical treatment for chronic sinusitis. Am J Rhinol 2002; 16: 303 312.

\author{
R.M. Naclerio \\ Department of Surgery, Section of \\ Otolaryngology-Head and Neck Surgery \\ The University of Chicago \\ Chicago, IL 60637 \\ USA \\ Tel: $+1-773-702-0080$ \\ Fax: +1-773-702-6809 \\ E-mail: rnacleri@surgery.bsd.uchicago.edu
}

